\title{
Vulnerabilidad derivada del trabajo productivo y reproductivo en secundarias
}

\section{Vulnerability derived from productive and reproductive work in middle schools}

\author{
Laura Gabriela Acosta Calderón \\ Servicios Educativos del Estado de Chihuahua \\ lauragacos@gmail.com \\ María Cristina Chávez Rocha \\ Centro de Investigación y Docencia \\ cristina.chavez@cid.edu.edu.mx \\ Argelia Antonia Ávila Reyes \\ Centro de Investigación y Docencia \\ argelia.avila@cid.edu.mx
}

\section{Resumen}

Esta investigación, formalizada en las escuelas secundarias en la ciudad de Chihuahua, tiene como objeto de estudio la vulnerabilidad derivada de los supuestos teóricos desde la perspectiva de género acerca del trabajo Productivo (remunerado) y Reproductivo (trabajo no remunerado de los hogares de las/los docentes, personal administrativo y de apoyo). Se desprende un proyecto institucional más amplio realizado en escuelas federalizadas en educación básica. Fue modificado el cuestionario de la Encuesta Nacional de uso del tiempo 2009 elaborada por el Instituto Nacional de Estadística y Geografía desde la perspectiva de género. De un total de 1200 instrumentos, 300 se aplicaron en secundaria. Debido a que este estudio se centra prioritariamente en determinar la vulnerabilidad derivada del trabajo Productivo y Reproductivo, se manejaron las dimensiones: Características socio demográficas, Toma de decisiones y, Actividades sociales, escolares y laborales. En los resultados se encontró que la vulnerabilidad humana (de la célula familiar, social, económica, derechos laborales), coincide con los roles y aspectos asignados por género prioritariamente los que desarrollan las mujeres.

\section{Palabras clave}

Vulnerabilidad, género, actividades del profesor, comportamiento, diferencias de género.

\begin{abstract}
This research, carried out in middle schools in the city of Chihuahua, has as object of study the vulnerability derived from the theoretical assumptions regarding gender perspective: Productive (paid) and Reproductive (unpaid work in households of the teachers, administrative and support staff). The study parts from a broader institutional project carried out in federalized schools in basic
\end{abstract}


education. The questionnaire of the National Survey on the use of time 2009 prepared by the National Institute of Statistics and Geography from a gender perspective was modified. Out of a total of 1200 instruments, 300 were applied in middle school. Because this study focuses primarily on determining the vulnerability derived from Productive and Reproductive work, the dimensions managed were: socio-demographic characteristics, decision-making and social, school and work activities. The results show that human vulnerability (in a nuclear family, social, economic, labor rights), matches those of the roles and aspects assigned by gender, primarily those developed by women.

\section{Keywords}

Vulnerability, gender, professor activities, behavior, gender differences.

\section{Introducción}

El objeto de estudio de la presente investigación es la vulnerabilidad por género derivada del trabajo productivo $\mathrm{y}$ reproductivo.

El trabajo reproductivo, llamado trabajo no remunerado de los hogares, no se le reconoce como trabajo, y permanece oculto sin valoración social ni económica. Esto debido a que lo realizan los miembros del hogar sin retribución monetaria y es esa falta de pago lo que origina que no se considere como una actividad productiva; por tanto, al no existir una transacción de mercado no se incluye en los principales índices económicos de nuestro país como es el Producto Interno Bruto (PIB).

Este tipo de labores domésticas se les distingue como funciones propias del género femenino, razón por la cual se ha impuesto a la mujer estos quehaceres como principal obligación. Adicionalmente, se cree que por ser mujer se tienen de forma innata las habilidades necesarias para la ejecución de las tareas del hogar y el cuidado de niños, adultos mayores y enfermos. Menoscabo de sus capacidades no se les permite el cultivo de su intelecto y la participación en el ámbito laboral. Es por ello la importancia de la transversalidad de la perspectiva de género para dicho estudio.

Se describen las condiciones a las que están expuestas/os los trabajadores de la educación en el nivel de secundarias. Considerar las posibilidades de que lo anterior, afecta a dichos trabajadores, en el aspecto económico, laboral y de género, creando una atmósfera de inestabilidad que perjudica su desempeño en todas las áreas.

\section{Antecedentes}

La desigualdad división sexual del trabajo es el factor explicativo de muchas discriminaciones que afectan a las mujeres. Hace más de una década que reflexionamos sobre la autonomía económica de las mujeres. En este sentido, sabemos que esa autonomía económica depende de la contribución de ellas a la creación de la riqueza a partir de su inserción en el mercado de trabajo a través del llamado trabajo productivo, aunque al mismo tiempo, del invisible trabajo reproductivo realizado en el ámbito doméstico.

Se percibe una contienda en México y en toda América Latina, entre la dinámica económica y de acumulación de capital por un lado, y la superación de las desigualdades, campo este donde se han logrado fuertes avances, a pesar de que debemos reconocer aún la persistencia del desafío de la superación. También dentro del campo de las políticas sociales hemos observado esa misma disociación, ya que tales políticas tomaron rumbos diversos entre la focalización y los avances distributivos hasta la continuidad de las políticas asistencialistas tradicionales. 
En México, el INEGI respetando los criterios internacionales elaboró durante el periodo 2006-2010 un estudio, sobre la cuenta satélite de trabajo no remunerado de los hogares de México, información sobre el tiempo destinado por las personas de 12 y más años de edad, a nivel nacional donde lanzó importantes cifras: se determinó que las horas de TNRH por semana para el 2010 fue de 1613 millones de hrs., por parte de las mujeres, $\mathrm{y}$ 417 millones de hrs. de los hombres.

De estas horas, la composición porcentual del TNRH fue: cuidados de salud el 14.3, ayuda y apoyo escolar el $21.8 \%$ y en cuidado general el $63.9 \%$, para el caso de las mujeres; y para el caso de los hombres se centra en cuidados de la salud.

\section{Planteamiento del problema}

La falta de reconocimiento del trabajo no remunerado dentro de las cuentas nacionales repercute negativamente sobre la igualdad de género en el nivel macroeconómico, debido a la importancia de estas cuentas como instrumentos para la formulación de políticas. En efecto, las cuentas nacionales cuantifican todas las áreas que se definen como parte de la economía nacional y, sobre esta base, se analizan la situación actual y las tendencias de la economía, se interpretan las dinámicas económicas y políticas $\mathrm{y}$, se hacen proyecciones sobre ellas, $\mathrm{y}$ se toman decisiones respecto a asignación de recursos.

El sentido del término "trabajo no remunerado de cuidado" el Fondo de Desarrollo de las Naciones Unidas para la Mujer (UNIFEM), define: "trabajo" enfatiza que la actividad tiene un costo en términos de tiempo y energía y que surge de una relación social, generalmente de carácter familiar, que se asume como obligación. "Cuidado" denota que la actividad se encamina a proporcionar servicios y bienestar a otras personas, en tanto que "no remunerado" resalta el carácter gratuito con que se cumple la actividad.
Mediante la siguiente pregunta, se buscó contestar cómo el personal puede estar expuesto a factores que lo vulneran dentro de su labor educativa, afectando su vida como un ser social, individual, haciendo una relación entre el estrés laboral y la salud para conocer las diferencias en función del género y del tipo de trabajo, los factores familiares, culturales además de lo individual:

¿En qué medida se manifiesta la vulnerabilidad por género derivada del trabajo productivo y reproductivo en las y los docentes, personal administrativo y de apoyo en secundarias?

Esta pregunta se fundamenta en las situaciones de reproductividad en tiempo y en economía, tomando en cuenta las situaciones de género y productividad que las mujeres pueden aportar $\mathrm{y}$, la vulnerabilidad que de esto pudiera derivarse. Para responder a las siguientes preguntas:

1. ¿Percibir menos ingresos, nos hace más vulnerables?

2. ¿Tener un nivel de estudios no profesional genera más vulnerabilidad?

3. ¿Es necesario dejar de ser vulnerable para cumplir con los trabajos productivo y reproductivo?

\section{Justificación}

El tema se abordó, respondió a las prioridades detectadas, que México se ha comprometido a asumir, a partir de la agenda acordada en la Cuarta Conferencia Mundial sobre las Mujeres, Beijing 1995, así como los seminarios internacionales de uso del tiempo, y las reuniones internacionales de estadística con perspectiva de género, desarrolladas en colaboración con ONU Mujeres, el Instituto Nacional de Estadística y Geografía (INEGI) y la Comisión Económica para América Latina y el Caribe (CEPAL); la Convención para la Eliminación de todas las Formas de Discriminación contra la Mujer 
(CEDAW,1972); el Programa Nacional para la Igualdad entre Mujeres y Hombres (PROIGUALDAD) y la Ley General para la Igualdad entre Mujeres y Hombres.

Además asume los avances que marcan un antes $y$ un después en la institucionalización de la perspectiva de género como política de Estado para mejorar la condición de los y las mexicanas. En primer lugar, la promulgación de la Ley General para la Igualdad entre Mujeres y Hombres y en segundo lugar, la promulgación de la Ley General de Acceso de las Mujeres a una Vida Libre de Violencia.

\section{Marco Teórico}

\section{Mujeres y su autonomía económica}

Las mujeres trabajan para su propio sustento y también el de otros ya hace mucho tiempo, su incorporación masiva al mercado de trabajo, ha sido un proceso relativamente reciente, fundamentalmente porque todas las tareas realizadas por las mujeres en el ámbito doméstico, implican una enorme magnitud de trabajo que genera riqueza y que sustenta y reproduce la vida en sociedad, este ámbito fue invisibilizado durante siglos y no considerado desde el punto de vista económico.

Tales trabajos, que incluyen actividades como preparar alimentos, buscar leña, llevar agua para dentro de la casa, mantener la limpieza de la casa, cuidar de la higiene familiar, cuidar de los ancianos, las y los niños, enseñarles a hablar y comportarse socialmente, asistir a las personas con necesidades especiales, y también a las y los enfermos, entre otras diversas tareas domésticas, imprescindibles para la vida y la socialización de las y los seres humanos. Lo que actualmente se denomina economía feminista ha incorporado la noción de esta contribución para la creación de riqueza por las sociedades.

Justamente por ese trabajo doméstico, no formar parte del mundo económico dentro del sistema capitalista centrado en la lógica del mercado, no asume ninguna transcendencia para los intercambios de valor, volviéndose así invisible, como también sin registrarse en las cuentas nacionales, y ni siquiera en la contabilidad doméstica.

\section{Trabajo productivo y reproductivo}

El concepto de trabajo es un concepto histórico, mucho se ha debatido sobre las maneras en que él encuentra en la base de la organización de las sociedades, e inclusive en las teorías económicas modernas y más ampliamente divulgadas, él está por detrás del precio de las mercaderías y del propio desarrollo de la humanidad.

Sin embargo, las consideraciones económicas, sociológicas, antropológicas y hasta filosóficas sobre el papel del trabajo y sus posibilidades de generar las condiciones de sobrevivencia de los seres humanos a través de la provisión de necesidades diversas en el tiempo y espacio, no llevaron en consideración el obvio valor del trabajo doméstico para justamente satisfacer las necesidades y carencias del cotidiano de la vida.

El trabajo productivo se refiere a aquel que genera mercaderías e ingreso, o sea, es aquel destinado a los intercambios del mercado, y que, al tener legitimación y valorización en ese mercado, es remunerado. La vulnerabilidad social es la que el ser humano vive desde que nace y puede ser impuesta deliberadamente o no, ya que son los únicos seres vivos que dependen durante los primeros cuatro años de vida de la madre y la familia para sobrevivir, a partir de esa edad sabrá por lo menos como hablar para externar sus necesidades, sin embargo, no son totalmente independientes.

\section{Vulnerabilidad en el trabajo}

"Un trabajo decente y productivo en condiciones de libertad, equidad, seguridad y 
dignidad humana", OIT (2009). Esta definición trae implícitos los cuatro objetivos estratégicos de dicha organización: el respeto de los derechos fundamentales en el trabajo, el acceso a un empleo digno, a la protección social y al diálogo social.

En la OIT, se manifiesta la idoneidad de lo que por norma debe ser un trabajo en el que no importan las características de la persona que lo desempeñe, sólo por el hecho de serlo debe tener esas garantías, es decir, el trabajar una jornada completa, si es su deseo, obtener el ingreso justo a esa actividad, según sus capacidades de estudio y/o habilidades y que además le permita satisfacer sus necesidades.

\section{Vulnerabilidad económica}

El término vulnerabilidad se ha convertido en un fértil instrumento de estudio de la realidad social, de disección de sus causas profundas, de análisis multidimensional que atiende no sólo a lo económico, como puede hacer la pobreza al menos en una visión clásica, sino también a los vínculos sociales, el peso político, el entorno físico y medioambiental o las relaciones de género, entre otros factores.

La vulnerabilidad económica está ligada a la vulnerabilidad de género debido al proceso histórico cultural generado entre el trabajo productivo y reproductivo donde este último podría ser reconocido como parte del desarrollo económico de la evolución social.

\section{Método}

Bajo el enfoque cuantitativo, lo que me permitió lograr una visión objetiva del tiempo empleado en hacer trabajo productivo reproductivo, ello fue posible a través de la estadística, característica de este método.

El alcance de la investigación es descriptivo. A través del estudio se conoce el número de horas empleadas por el personal de las secundarias encuestadas para hacer su trabajo productivo, el trabajo reproductivo; es de carácter exploratorio ya que como objeto de estudio ha sido poco estudiado, por lo que se hizo desde una perspectiva innovadora que preparara el terreno para nuevos estudios y/o posibles soluciones.

El diseño de la investigación es de tipo no experimental, transversal cuyo propósito fue la recolección de datos en un único momento mediante la aplicación de cuestionarios, de tipo exploratorio y descriptivo.

Se aplicó un cuestionario, para obtener el resultado de su salario al personal de dicha dependencia y el tiempo empleado normalmente para realizar trabajo reproductivo, esto es, actividades personales, familiares y sociales que van desde la preparación y el servicio de los alimentos para los integrantes del hogar, la limpieza de la vivienda, limpieza y cuidado de la ropa y calzado, mantenimiento, instalación y reparación a la vivienda y a los bienes del hogar, compras, administración del hogar, asistencia a eventos culturales, deportivos y de entretenimiento, utilización de medios de comunicación, cuidados personales, traslado de la familia, visitas al médico, tareas de los hijos, entre otras cosas.

\section{Técnica de investigación}

El método empleado fue la encuesta, se eligió como técnica el cuestionario. El cuestionario fue modificado por el grupo de maestría, tomando en cuenta la perspectiva de género, en donde nuestras asesoras del grupo fungieron como especialistas en el tema.

Dicho instrumento ya modificado, fue piloteado en el mes de enero de 2013, en dos momentos, con quince días de diferencia en escuelas similares a la muestra, en los subsistemas de Educación Preescolar, Primaria y Secundaria, arrojando un coeficiente de correlación de Pearson arrojando un grado de confiabilidad de 0.74, es decir con una correlación positiva 
considerable calculada a partir de las puntuaciones obtenidas en el pilotaje.

El cuestionario está integrado con cuatro dimensiones, 20 secciones y 160 reactivos, fue validado en una muestra de 208 personas seleccionadas a través del programa Random, el cual señaló la cantidad de instrumentos a aplicar después de ingresados los datos. Con este programa se buscó que el margen de error fuera pequeño y la confianza lo máximo a través del STATS que nos dio el error máximo aceptable que es del 1 al 5\% para lograr el nivel deseado de confianza.

\section{Población y muestra}

Inicialmente se contempló la aplicación a una muestra aleatoria de 5000 instrumentos, pero dado el bajo presupuesto fueron autorizados por la dirección del Centro de Investigación y Docencia (CID), un total de 1200 instrumentos, de los cuales 300 se aplicaron a secundarias.

\section{Variables}

Características Socio demográficas
Comportamiento humano
Trabajo
Libertad
Decidir
Permisos
Relaciones Sexuales
Anticonceptivos

\section{Indicadores}

Los ítems o mediciones coherentes con las variables, y se tomaron en cuenta para la elaboración de las preguntas en el cuestionario:

Medición de la vulnerabilidad derivada del trábalo productivo y reproductivo del personal de secundarias federalizadas:
CARACTERÍSTICAS SOCIO- DEMOGRÁFICAS (Parentesco con el jefe (a) del hogar, sexo, edad, nivel de estudios, estado civil)

Indicadores: sexo, edad, estatus familiar escolaridad, dominio laboral COMPORTAMIENTO HUMANO: Trabajo, libertad, decidir, permisos, relaciones sexuales, anticonceptivos. Indicadores: Resolución que se toma o se da en una cosa ante las que existen dos o más alternativas.

Algunos resultados

Alcance exploratorio

Características socio demográficas: Del 100\% de los encuestados hombres, se observa que el $20 \%$ tiene maestría, el $44 \%$ cuenta con estudios profesionales, otro $20 \%$ tiene bachillerato, $4 \%$ normal básica, $4 \%$ carrera técnica, $4 \%$ secundaria, $4 \%$ no contestó. En caso de las mujeres encuestadas, el $17 \%$ tiene maestría, un $2.8 \%$ cuenta con doctorado, el $34 \%$ tiene estudios profesionales (normal superior, licenciatura, ingeniería), el 4\% normal básica, $18 \%$ con carrera técnica, el $21 \%$ con bachillerato.

Edad: La edad de los y las encuestadas varia en su mayoría de entre los 27 a los 55, de mayor a menor porcentaje fue de entre los 4655 con un $43.2 \%$, un $21.1 \%$ de 35 a 45 y un $17.9 \%$ de $27-35$ años.

Se ve más participación a colaborar expresando las opiniones entre las personas de 35 a 55 años, donde se considera un rango de edad de madurez.

Puesto de trabajo-Sexo-Edad: se observa que las mujeres docentes $y$ administrativas de 45 a 55 años son mayoría con el $28 \%$, del total de la muestra, en segundo lugar están las mujeres docentes y las administrativas de 35-45 años con el 14\%, les siguen las mujeres docentes y administrativas de 27-35 años con un 12\%, siendo estas las edades que predominan y siendo mujeres un $54 \%$ del total de la muestra y en los puestos ya mencionados.

Sexo-Estado civil: el $67 \%$ del total de encuestados y encuestadas, son mujeres casadas, el $68 \%$ son hombres casados. 
De los datos en general el 10\% soltero (a), 8\% divorciada (o), 67\% casada (o) en esta tabla se arrojan datos culturales donde por obtención de status la mujer en el modelo judeo cristiano que nos caracteriza como sociedad la mujer deben estar casada y ser compañera y está convencida de ello según se constata en la tabla siguiente: ¿Qué parentesco tiene con el jefe del hogar?

\section{Reflexiones Finales}

La vulnerabilidad económica se manifiesta en los Docentes, personal administrativo $\mathrm{y}$ personal de apoyo, mediante la exposición de las políticas públicas que derivan en la Reforma Educativa que hasta la fecha, impacta en las contrataciones temporales y en las evaluaciones a los directivos, y docentes ( por lo pronto), que hace que la estabilidad de pertenecer al gremio educativo, genere incertidumbre, por depender de los resultados de una evaluación al desempeño, basada en el "deber ser", y no, en la contextualización del entorno del lugar y de las condiciones de trabajo.

La vulnerabilidad económica en el trabajo reproductivo, implica no ingresos, y se une la vulnerabilidad de género, basados en el modelo judeo cristiano, donde las actividades de este tipo, se realizan por asignación al género femenino, y la retribución es ser mantenida, en el caso del personal involucrado en este trabajo, se suma al de cumplir con las actividades laborales para los que fueron contratados, y llegar a casa a continuar trabajando en las labores de casa y cuidado de la familia.

Las mujeres vulnerables se caracterizan por una actitud sumisa que les lleva a dejar de tomar decisiones, desarrollando en su mayoría una codependencia hacia la presencia masculina (padres, hermanos, esposo, hijos, jefes), aunque en ellos encuentre vejaciones, desprecios, maltratos o manipulación (emocional, o económica). Asumen el rol de abnegación y sumisión que invade todos los ámbitos de su vida, ante los abusos laborales, las cuestiones de vida marital y sexual.

De acuerdo a los resultados, se asume que la vulnerabilidad derivada del trabajo productivo y reproductivo en secundarias es alta, dado que los docentes, personal de apoyo y administrativo de esta área, están sujetos a factores como el Burnout, discriminación por sexo, falta de oportunidades profesionales y estancamiento laboral, una reforma denominada educativa, que presenta mayores elementos de reforma laboral, la cual anula la estabilidad que caracteriza al sector educativo y que por lo tanto lo vulnera.

\section{Referencias}

Aguilar, L. Mercedes y Espinoza, I. 1998. Informe Uso del Tiempo Nicaragua. Gobierno de la República de Nicaragua. Instituto nacional de estadísticas y censos.

Benería, L. 1999. El debate inconcluso sobre el trabajo no remunerado Organización Internacional del trabajo (OIT, 1999). Revista Internacional del trabajo, vol. 118, núm. 3.

Benería, L. $2006 . \quad$ Trabajo productivo/reproductivo, pobreza y políticas de conciliación. Nómadas (Col), Núm. 24, pp. 8-21, abril.

CEPAL-ECLAC, Vulnerabilidad Socio demográfica: viejos y nuevos riesgos para comunidades hogares y personas. Brasilia, Brasil, ONU, LC/R.2086

Fuentes M. Molinar. 1986. Crítica a la escuela: el reformismo radical en Estados Unidos. Antología. Biblioteca Pedagógica, Mex. D.F. Essay on education, Jules Henry, penguin.1971.

Fuentes R. 2010. Las condiciones laborales y profesionales de los docentes de las escuelas secundarias. Centro de Investigación y Docencia 
Gammage, S. 2009. Género, pobreza de tiempo y capacidades en Guatemala: Un análisis multifactorial desde una perspectiva económica. CEPAL.
Garavito, C. 2006. Vulnerabilidad en el Empleo: Género y Etnicidad, Departamento de Economía de la Pontificia Universidad Católica del Perú. 